\title{
High catechin concentrations detected in Withania somnifera (ashwagandha) by high performance liquid chromatography analysis
}

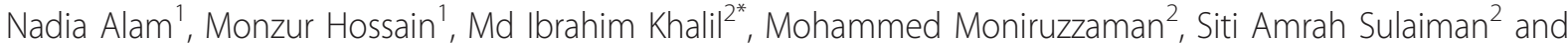
Siew Hua Gan ${ }^{3}$

\begin{abstract}
Background: Withania somnifera is an important medicinal plant traditionally used in the treatment of many diseases. The present study was carried out to characterize the phenolic acids, flavonoids and 1,1-diphenyl-2picrylhydrazyl radical (DPPH) scavenging activities in methanolic extracts of $W$. somnifera fruits, roots and leaves (WSFEt, WSREt and WSLEt).

Methods: WSFEt, WSREt and WSLEt was prepared by using $80 \%$ aqueous methanol and total polyphenols, flavonoids as well as DPPH radical scavenging activities were determined by spectrophotometric methods and phenolic acid profiles were determined by HPLC methods.

Results: High concentrations of both phenolics and flavonoids were detected in all parts of the plant with the former ranging between $17.80 \pm 5.80$ and $32.58 \pm 3.16 \mathrm{mg} / \mathrm{g}$ (dry weight) and the latter ranging between $15.49 \pm$ 1.02 and $31.58 \pm 5.07 \mathrm{mg} / \mathrm{g}$. All of the three different plant parts showed strong DPPH radical scavenging activities $(59.16 \pm 1.20$ to $91.84 \pm 0.38 \%)$. Eight polyphenols (gallic, syringic, benzoic, p-coumaric and vanillic acids as well as catechin, kaempferol and naringenin) have been identified by HPLC in parts of the plant as well. Among all the polyphenols, catechin was detected in the highest concentration (13.01 \pm 8.93 to $30.61 \pm 11.41 \mathrm{mg} / \mathrm{g}$ ).

Conclusion: The results indicating that $W$. somnifera is a plant with strong therapeutic properties thus further supporting its traditional claims. All major parts of $W$. somnifera such as the roots, fruits and leaves provide potential benefits for human health because of its high content of polyphenols and antioxidant activities with the leaves containing the highest amounts of polyphenols specially catechin with strong antioxidant properties.
\end{abstract}

Keywords: Withania somnifera, spectrophotometry, HPLC, polyphenols, antioxidant, free radical scavenging activity

\section{Background}

Ashwagandha [Withania somnifera L. Dunal] (Solanaceae) is an important medicinal plant, commonly-used as a domestic remedy for several diseases in India as well as other parts of the world [1]. It is described as an herbal tonic and health food in the famous book of Vedas and is considered as in 'Indian Ginseng' in traditional Indian system of healing [2]. Several recent reports have demonstrated immunomodulator and antitumor effect of W. somnifera as well [3]. Moreover,

\footnotetext{
* Correspondence: mibrahim12@yahoo.com

${ }^{2}$ Department of Pharmacology, School of Medical Sciences, Universiti Sains

Malaysia, 16150 Kubang Kerian, Kelantan, Malaysia Full list of author information is available at the end of the article
}

various parts of the plant have been reported to possess antiserotogenic, anticancer and anabolic properties and have shown beneficial effects in the treatment of arthritis, stress and geriatric problems [4]. W. somnifera is also made into dietary supplements with good nutritional properties and phytochemicals. Besides being used among the elderly to increase health vitality, a decoction of W. somnifera root is also used as nutrient and health restorative agent among postpartum ladies as it was purported to thicken and increase the nutritive value of the breastmilk when given to nursing mothers. The pharmacological effect of the roots of W. somnifera is attributed to its active ingredient, withanolides [5] which has a wide range of therapeutic applications [6].
C Biomed Central 
There is a great deal of evidence indicating that excessive free radical production and lipid peroxidations are actively-involved in the pathogenesis of a wide number of chronic diseases, including atherosclerosis [7], cardiac and cerebral ischemia [8], neurodegenerative disorders [9], carcinogenesis [10], diabetes [11] and rheumatic disorders [12] and contributes a major role in the ageing process [13]. Plant-derived antioxidants such as vitamin E, vitamin $C$, polyphenols including phenolic acids, phenolic diterpenes, flavonoids, catechins, procyanidins and anthocyanins are becoming increasingly important as dietary factors [14]. Supplementation with berry juice [15], flavones from skullcap, catechins from green tea, anthocyanins from chokeberry and condensed tannins from fava beans [16] are indices of oxidative stress protectant in rats. Furthermore, the growing interest in the substitution of synthetic food antioxidants by natural chemicals has fostered research on plant sources and the screening of raw materials for identifying new antioxidants. In this regard, polyphenols are being increasingly reported to exhibit antioxidant effects in foods [17]. Various plants have been analysed for the existence of phenolic acids by HPLC [18]. Plant acids are known to have anticarcinogenic activity [19]. and phenolic compounds are believed to be an important part of the general defence mechanism of many plants against infections [20]. Therefore, it is useful to measure the presence of phenolic compounds in natural substances.

Purification of phenolic acids is very difficult not only due to their isomeric similarities but also due to the influence of various effects such as acid-based treatment, temperature and light on their labile structures [21]. The determination of phenolic acids is important both for their characterization and to facilitate more efficient uses of important plant resources [22].

To date, very limited data exists on phenolic compounds reported in W. somnifera leaves, roots and fruits as well as their antioxidant effects to support their traditional claims. Therefore, we aimed to undertake this task in the present study as W. somnifera is widely-used. If the presence of phenolic and flavonoid compounds present in $W$. somnifera can be confirmed, the plant can be used as a good possible source of antioxidant.

\section{Methods}

\section{Plant material}

The W. somnifera plant parts such as roots, fruits and leaves were collected from field grown plants after six months of cultivation in Botanical Garden, Rajshahi University, Bangladesh. The collected parts of medicinal plant were brought into the laboratory, cleaned and airdried in shade and then grinded to a fine powder.

\section{Chemicals and reagents}

Reagents such as 1,1-diphenyl-2-picrylhydrazyl radical (DPPH) and Folin-Ciocalteu's reactive were purchased from Sigma-Aldrich (St. Louis, USA). Sodium carbonate $\left(\mathrm{Na}_{2} \mathrm{CO}_{3}\right)$, aluminium chloride $\left(\mathrm{AlCl}_{3}\right)$, sodium nitrite $\left(\mathrm{NaNO}_{2}\right)$ and sodium hydroxide $(\mathrm{NaOH})$ were purchased from Merck (Darmstadt, Germany). All chemicals used were of analytical grades.

\section{Preparation of Plant Extracts}

W. somnifera roots, fruits and leaves extract (WSREt, WSFEt and WSLEt) preparation was performed according to a modified method described by Kahkonen et al., [23]. Grinded dry plant materials (500 mg) were weighed into a test tube followed by the addition of a total of $10 \mathrm{ml}$ of $80 \%$ aqueous methanol. The suspension was then stirred slightly. The tubes were sonicated for $5 \mathrm{~min}$ and centrifugated for another $10 \mathrm{~min}$ at 1500 $\mathrm{g}$ and the resulting supernatants were collected. The extraction procedure was repeated and the supernatants were combined before being evaporated to a volume of approximately $1 \mathrm{ml}$. The concentrated extracts were further lyophilized and weighed.

\section{Total Polyphenols}

Phenolic compounds in W. somnifera were estimated by a spectrophotometric determination using a modified Folin-Ciocalteu method Singleton et al., [24]. Briefly, $100 \mu \mathrm{l}$ of sample extracts $(1 \mathrm{mg} / \mathrm{ml})$ was mixed with 1 $\mathrm{ml}$ of Folin and Ciocalteu's phenol reagent $(2 \mathrm{~N})$. After 3 minutes, $1 \mathrm{ml}$ of $10 \% \mathrm{Na}_{2} \mathrm{CO}_{3}$ solution was added to the mixture and adjusted to $10 \mathrm{ml}$ with distilled water. The reaction was kept in the dark for $90 \mathrm{~min}$, after which the absorbance was read at $725 \mathrm{~nm}$ (T $80 \mathrm{UV} /$ VIS spectrophotometer, ChromoTek GmbH, Germany). Gallic acid was used to calculate the standard curve (20, $40,60,80$ and $\left.100 \mu \mathrm{g} / \mathrm{ml}, \mathrm{r}^{2}=0.993\right)$. Estimation of the phenolic compounds was carried out in triplicates. The results were mean values \pm standard deviations and expressed as milligrams of gallic acid equivalents (GAEs) per g of W. somnifera dry weight (DW).

\section{Determination of total flavonoids}

The total flavonoid contents of the $W$. somnifera extracts were determined according to the colorimetric assay method developed by Zhishen et al., [25]. Briefly, $1 \mathrm{ml}$ of properly diluted $(1 \mathrm{mg} / \mathrm{ml})$ WSREt, WSFEt and WSLEt were mixed with $4 \mathrm{ml}$ of distilled water. At baseline, $0.3 \mathrm{ml}$ of $(5 \% \mathrm{w} / \mathrm{v}) \mathrm{NaNO}_{2}$ was added. After five minutes, $0.3 \mathrm{ml}$ of $(10 \% \mathrm{w} / \mathrm{v}) \mathrm{AlCl}_{3}$ was added followed by the addition of $2 \mathrm{ml}$ of $\mathrm{NaOH}$ solution ( $1 \mathrm{M})$ six minutes later. After that, the volume was immediately made up to $10 \mathrm{ml}$, with the addition of $2.4 \mathrm{ml}$ of distilled water. The mixture was shaken vigorously and the 
absorbance of the mixture was read at $510 \mathrm{~nm}$. A calibration curve was prepared using a standard solution of catechin $\left(20,40,60,80\right.$ and $\left.100 \mu \mathrm{g} / \mathrm{ml}, \mathrm{r}^{2}=0.996\right)$. The results were expressed as $\mathrm{mg}$ catechin equivalents (CEQ) per g of $W$. somnifera (DW).

\section{DPPH free radical-scavenging activity}

The antioxidant capacity of the $W$. somnifera was also studied through the evaluation of the free radicalscavenging effect on the DPPH radical. The determination was based on the method proposed by Ferreira et al., [26]. Briefly, $1 \mathrm{ml}(1 \mathrm{mg} / \mathrm{ml})$ of WSREt, WSFEt and WSLEt were mixed with $2.7 \mathrm{ml}$ of methanolic solution containing DPPH radicals $(0.024 \mathrm{mg} / \mathrm{ml})$. The mixture was vigorously shaken and left to stand for $60 \mathrm{~min}$ in the dark (until their absorbance remained unchanged). The reduction of the DPPH radical was determined by measuring the absorbance at $517 \mathrm{~nm}$ [27]. The radicalscavenging activity (RSA) was calculated as a percentage of DPPH discolouration using the equation: \% RSA = $\left[\left(A_{D P P H}-A_{S}\right) / A_{D P P H}\right] \times 100$, where $A_{S}$ is the absorbance of the solution when the sample extract has been added at a particular level and $\mathrm{A}_{\mathrm{DPPH}}$ is the absorbance of the DPPH solution.

\section{High Performance Liquid Chromatography (HPLC)}

The HPLC method was based on the method published by Kaškonienè et al., [28]. Analysis of WSREt, WSFEt and WSLEt were performed by employing an HPLC system (Waters 2695, Milford, MA, USA) equipped with a Photodiode Array Detector (Waters 2996, Milford, MA, USA). The HPLC column was a Merck Purospher Star, RP-18e, $(125 \times 4 \mathrm{~mm}, 5 \mu \mathrm{m})$ fitted with a guard cartridge packed with the same type of stationary phase (Merck, Darmstadt, Germany). The linear 76 gradient was used at a flow rate of $0.5 \mathrm{ml} / \mathrm{min}$ with total analytical time of approximately $35 \mathrm{~min}$. The binary mobile phase consisted of a solvent A (ultra pure water with $0.1 \%$ of phosphoric acid) and solvent B (pure methanol with $0.1 \%$ of phosphoric acid). Elution from the column was achieved with the following gradient: $0 \mathrm{~min}$ to 10 min of solvent $B$, increased from $35 \%$ to $55 \%$; $10-25 \mathrm{~min}$ of solvent B, increased to $62 \%$; $25-30$ min of solvent B, increased to $85 \%$ and the final composition was kept constant till $35 \mathrm{~min}$. All solvents used were of HPLC grade quality. The detection wavelength was done between 200 and $450 \mathrm{~nm}$ with specific monitoring at $265 \mathrm{~nm}$. The identification of phenolic compounds was performed by comparing the retention time and UV absorption $\left(\lambda_{\max }\right)$ of each peak of the analytes with the reference standards. Phenolic acids (gallic, syringic, caffeic, vanillic, p-coumaric, benzoic and transcinnamic acids) as well as flavonoids (catechin, naringenin, luteolin, hesperetin, kaempferol, apigenin, naringin) were purchased from Sigma (St. Louis, MO, USA) and were used as reference standards.

\section{Statistical analysis}

All analyses were carried out in triplicates and the data was expressed as means \pm standard deviations (SD). The data was analyzed using (Statistical Packages for Social Science 12.0) (SPSS Inc., USA) and MS Excel 2003. One-way analysis of variance (ANOVA) followed by Tukey's honestly significant difference post hoc test was used to compare the phenol contents, FRAP values, DPPH scavenging activities and colour parameters of WSREt, WSFEt and WSLEt (Table 1). The differences between means at $95 \%(\mathrm{p}<0.05)$ confidence level were considered statistically significant. Correlations were obtained by Pearson's correlation coefficient (r) in bivariate linear correlations.

\section{Results}

\section{Phenolic content}

The contents of total polyphenols (mg GAE/g) of WSREt, WSFEt and WSLEt were investigated using the modified Folin-Ciocalteu assay which is sensitive to phenol and polyphenols entities and other electron donating antioxidants such as ascorbic acid and vitamin E. The sources of the analysed WSREt, WSFEt as well as WSLEt were significantly different $(\mathrm{p}<0.05)$, as shown in (Table 1). Among the three different W. somnifera extracts, the concentrations of polyphenols was found to be lowest in WSREt $(17.80 \pm 5.80 \mathrm{mg} / \mathrm{g})$ and highest in WSLEt $(32.58 \pm 3.16 \mathrm{mg} / \mathrm{g})$.

\section{Flavonoids content}

The total contents of flavonoids of the three different W. somnifera extracts were also determined. Flavonoids were detected in high concentrations ranging from $15.49 \pm 1.02$ (WSREt) to $31.58 \pm 5.07$ (WSLEt) $\mathrm{mg}$ CEQ/g (Table 1).

\section{DPPH radical scavenging activity}

There were significant differences in terms of their scavenging abilities present among the WSREt, WSFEt as well as WSLEt samples, expressed as percentage of inhibition on the DPPH radical (Table 1). Among the three extracts, the lowest scavenging activity was found in WSREt $(59.16 \pm 1.20 \%)$ while the highest activity was found in WSLEt $(91.84 \pm 0.38 \%)$. The DPPH radical scavenging test is one of the fastest tests available to investigate the overall hydrogen/electron donating activity of single antioxidants and health-promoting dietary antioxidant supplements. The reasons behind the markedly higher radical scavenging capacity exhibited by the different types of $W$. somnifera extracts probably lie in their diverse botanical origin. Antioxidant potential of 
Table 1 Spectrophotometric analysis of phenolics, flavonoids and antioxidant properties of W. somnifera roots, fruits and leaves.

\begin{tabular}{lccc}
\hline W. somnifera & Phenolics mg GAE/g (DW) & Flavonoids mg CEQ/g (DW) & \% of DPPH inhibition \\
\hline Roots & $17.80 \pm 5.80^{c}$ & $15.49 \pm 1.02^{c}$ & $59.16 \pm 1.20^{c}$ \\
Fruits & $22.29 \pm 1.99^{\mathrm{b}}$ & $21.15 \pm 5.32^{\mathrm{b}}$ & $70.38 \pm 0.84^{\mathrm{b}}$ \\
Leaves & $32.58 \pm 3.16^{\mathrm{a}}$ & $31.58 \pm 5.07^{\mathrm{a}}$ & $91.84 \pm 0.38^{\mathrm{a}}$ \\
\hline
\end{tabular}

In each column, values with different letters (superscripts) indicate significant differences $(p<0.05)$. DW $=$ dry weight

W. somnifera extracts is directly related to its phenolic and flavonoids content.

\section{Correlations}

The correlations among the phenolic compounds, flavonoids and DPPH radical scavenging activities are shown in (Table 2). The correlation matrix showed that significant linear correlation exists between the results of all three analytical methods employed indicating that the three measurements are reliable indicators of antioxidant activities. The lowest linear correlation value at $\mathrm{r}=$ $0.962(\mathrm{p}=0.01)$ and the highest correlation value at $\mathrm{r}=$ 0.995 . Both phenolic compounds and DPPH radical scavenging activity are strongly correlated $(r=0.995$ and 0.983 respectively) with DPPH radical scavenging activities. The significant correlations existing between phenolic compounds and DPPH radical scavenging activities indicate the strong antioxidant properties of the tested WSREt, WSFEt and WSLEt. Similar to our findings, some literature also reported strong correlation between the antioxidant capacity and total phenolic contents [29] further suggesting that polyphenols are the major components responsible for the antioxidant effects of WSREt, WSFEt and WSLEt.

\section{HPLC analysis}

Sixteen phenolic and flavonoid standards were compared with the chromatograms produced by the unknown W. somnifera extracts. HPLC analysis of phenolic and flavonoids compounds in WSREt, WSFEt as well as WSLEt showed that only catechin is commonly found in all of the three extracts analyzed. Eight polyphenols (five phenolic acids and three types of flavonoids) have been identified and the phenolics patterns of all plant parts were confirmed to contain gallic,

Table 2 Correlations matrix among phenolics, flavonoids content and free radical scavenging activities Correlations

\begin{tabular}{lccc}
\hline & phenolics & Flavonoids & DPPH \\
\hline phenolics & 1 & $\left.0.995^{(* *}\right)$ & $\left.0.983^{(* *}\right)$ \\
Flavonoids & $\left.0.995^{(* *}\right)$ & 1 & $\left.0.962^{(* *}\right)$ \\
DPPH & $\left.0.983^{(* *}\right)$ & $0.962^{(*)}$ & 1 \\
\hline
\end{tabular}

** Correlation is significant at the 0.01 level (2-tailed). syringic, benzoic, p-coumaric and vanillic acids as well as the flavonoids catechin, kaempferol and naringenin.

(Figures 1, 2, 3) show the HPLC chromatograms obtained from WSREt, WSFEt and WSLEt. Six phenolic compounds were detected in WSLEt whereas, three compounds were identified in WSFEt and only two compounds were identified in WSREt. The unknown compounds that may have had similar flavonoid and phenolic acid spectra and chromatographic behaviours (shown as extra peaks in the figures) were also detected. However, they could not be fully identified due to lack of standard compounds. (Figure 4) compared the total phenolic compounds of sample extracts obtained when using spectrophotometric and HPLC methods. Overall, spectrophotometric methods tend to report higher levels of phenolics when compared to HPLC method.

\section{Discussion}

To our knowledge, our study is the first to identify phenolic compounds present in W. somnifera. The present study confirmed the presence of phenolic compounds, flavonoids and antioxidant activities in WSREt, WSFEt and WSLEt. High concentrations of phenolic compounds were found in the different parts of W. somnifera with significant variations in the amount. (Table 1) showed high content of phenolics and flavonoids in WSLEt $(32.58 \pm 3.16$ and $31.58 \pm 5.07$ respectively) while that in WSREt was low $(17.80 \pm 5.80$ and $15.49 \pm$ 1.02 respectively). This indicates that the leaves of $W$. somnifera should be consumed for its antioxidant effects. Several reports have shown that different plant parts have variable polyphenols compositions, as shown by our findings [30].

There is very poor data on analysis of phenolic compounds in W. somnifera. Udayakumar et al., [30] from India reported that the presence of total phenolic compounds in WSREt was $28.26 \mathrm{mg} / \mathrm{g}$ while that of flavonoids was $17.32 \mathrm{mg} / \mathrm{g}$. For WSLEt it was $5.4 \mathrm{mg} / \mathrm{g}$ total phenolic compounds and $5.1 \mathrm{mg} / \mathrm{g}$ flavonoids both of which were different from our study perhaps due to the different source of W. somnifera and polyphenols of plant parts which may also be related to the colour, maturity and environment. However, the spectrophotometric method tend to overestimate the phenolics content with respect to the chromatographic method 


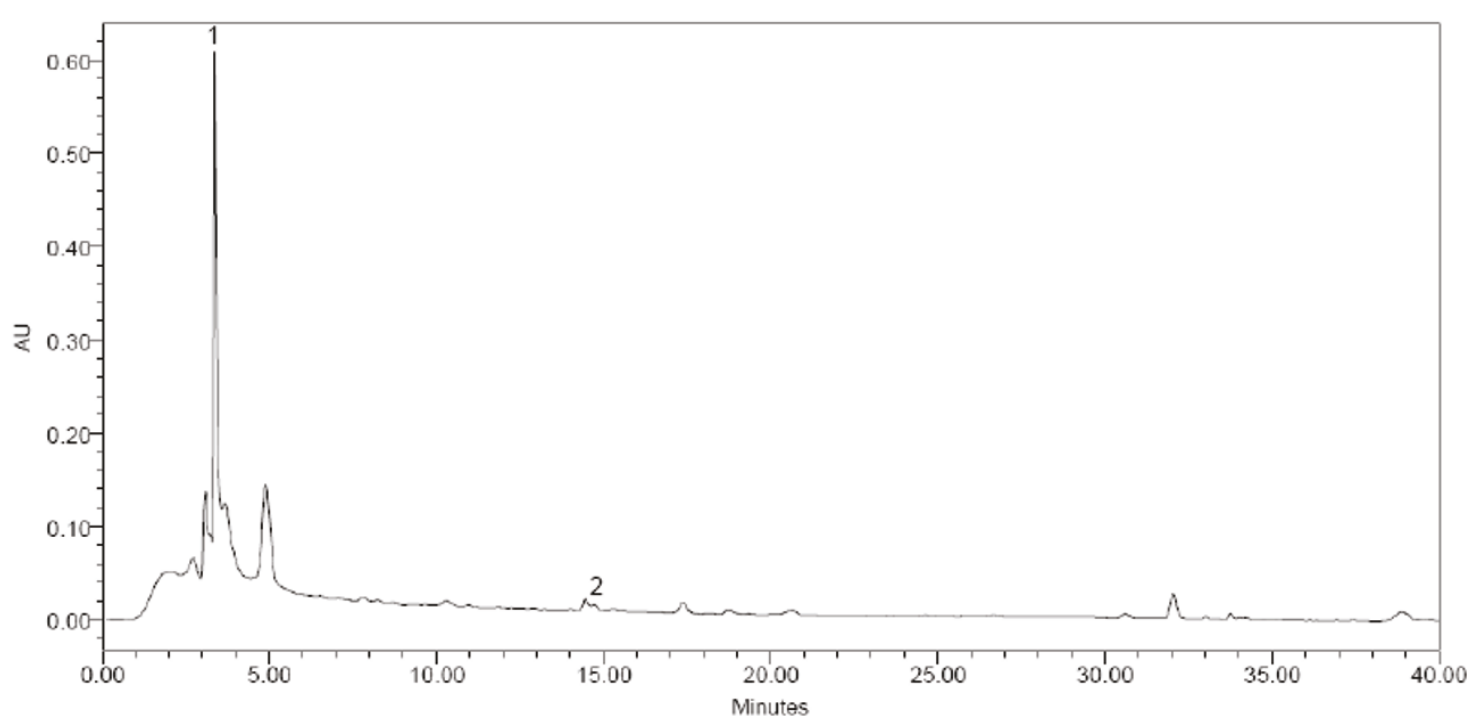

Figure 1 HPLC chromatogram of $\boldsymbol{W}$. somnifera roots. (1) catechin and (2) benzoic acid.

perhaps due to the fact that non-phenolic materials present in the investigated extracts interfered in the spectrophotometric analysis [31].

The scavenging ability of DPPH free radical is extensively used to screen the antioxidant potential of naturally-derived foods and plants. Methanol was employed in this study to extract the low molecular weight and moderately polar substances because of its wide solubility properties. We found that WSREt, WSFEt and WSLEt exhibited free radical DPPH scavenging abilities (Table 1). In this study, we attempted to isolate the active compounds responsible for antioxidant activities in WSREt, WSFEt and WSLEt. The antioxidant capacity has been shown to be directly related with the total phenolic content (Table 2) which was in agreement with many previous reports [32-34]. In addition, the flavonoids contributed to almost all of the total phenolic content (Table 1), which indicated that the flavonoids in W. somnifera are important constituents responsible for the bioactivities.

In HPLC analysis, six compounds were identified in WSLEt while three were identified in WSFEt and two were identified in WSREt. Out of the eight phenolic compounds catechin was found in the highest

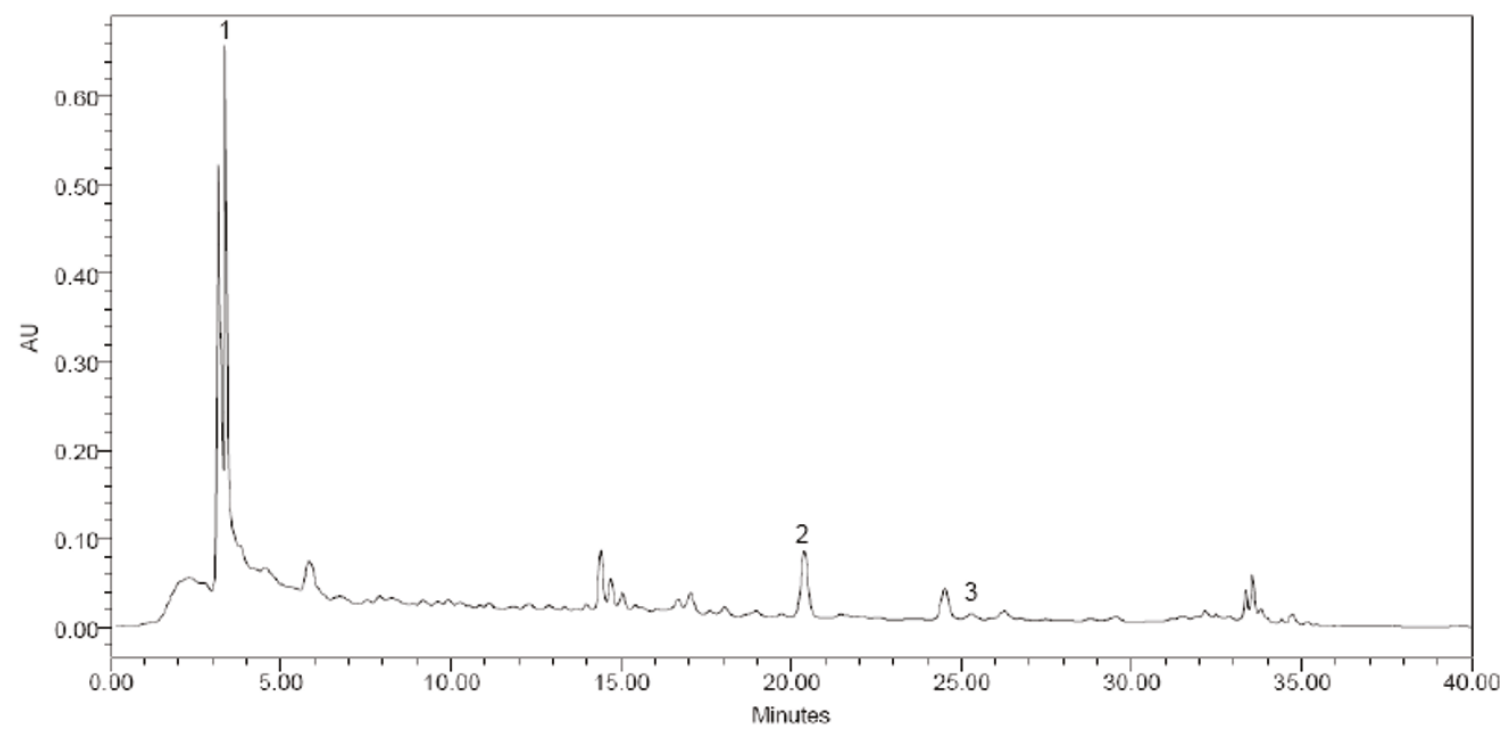

Figure 2 HPLC chromatogram of $\boldsymbol{W}$. somnifera fruits. (1) catechin (2) naringenin and (3) kaempferol. 


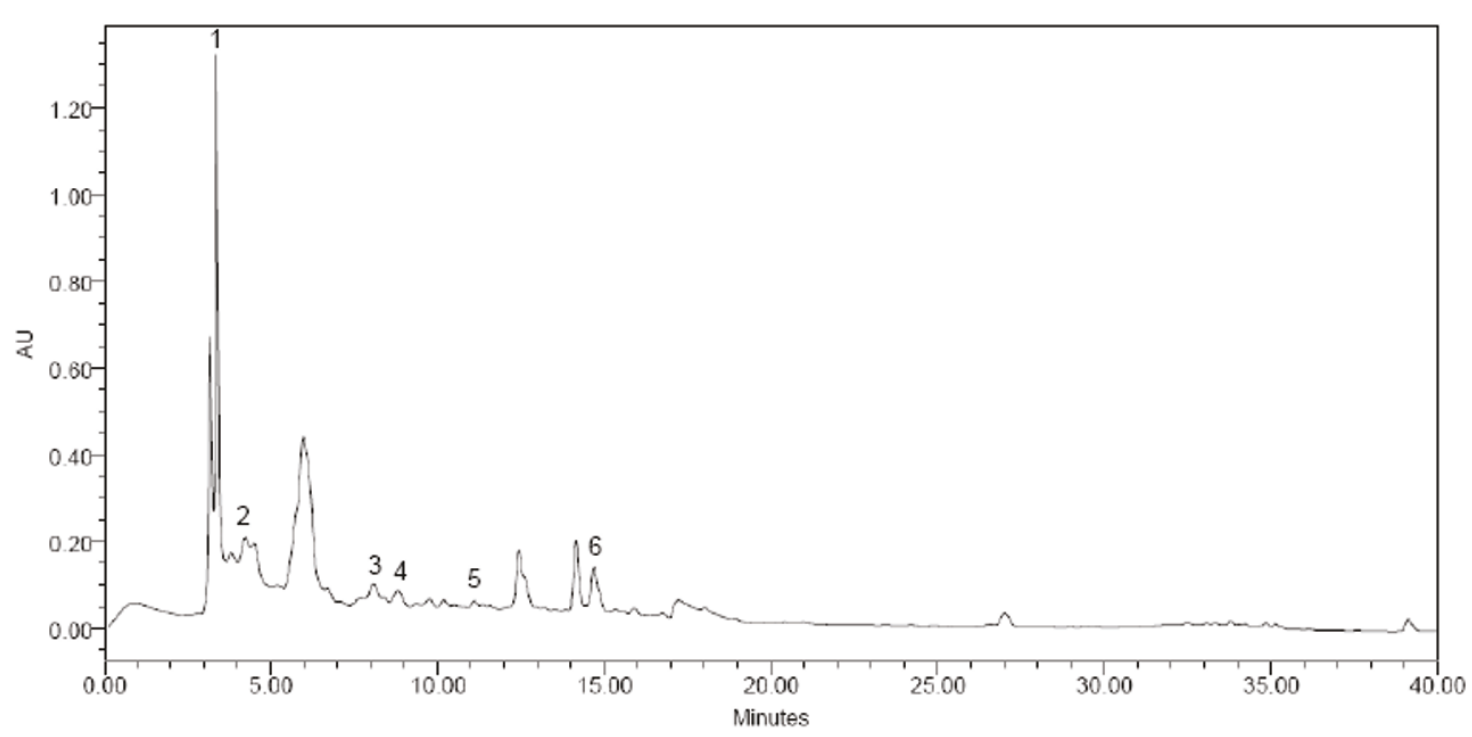

Figure 3 HPLC chromatogram of $\boldsymbol{W}$. somnifera leaves. (1) catechin, (2) gallic acid, (3) syringic acid, (4) vanillic acid, (5) p-coumaric acid and (6) benzoic acid.

concentration compared to others amounting $12.82 \mathrm{mg} /$ $\mathrm{g}$ in WSREt, 19.48 in WSFEt and $28.38 \mathrm{mg} / \mathrm{g}$ in WSLEt (Table 3). This indicates that WSREt, WSFEt and WSLEt are rich sources of catechin. Catechin is one of the most important polyphenols that provide health benefits and is found in high quantities in green tea which is widely known for its strong antioxidant properties. There are many reports on catechin which described its therapeutic role in human health. Modern studies have found that catechin is responsible for

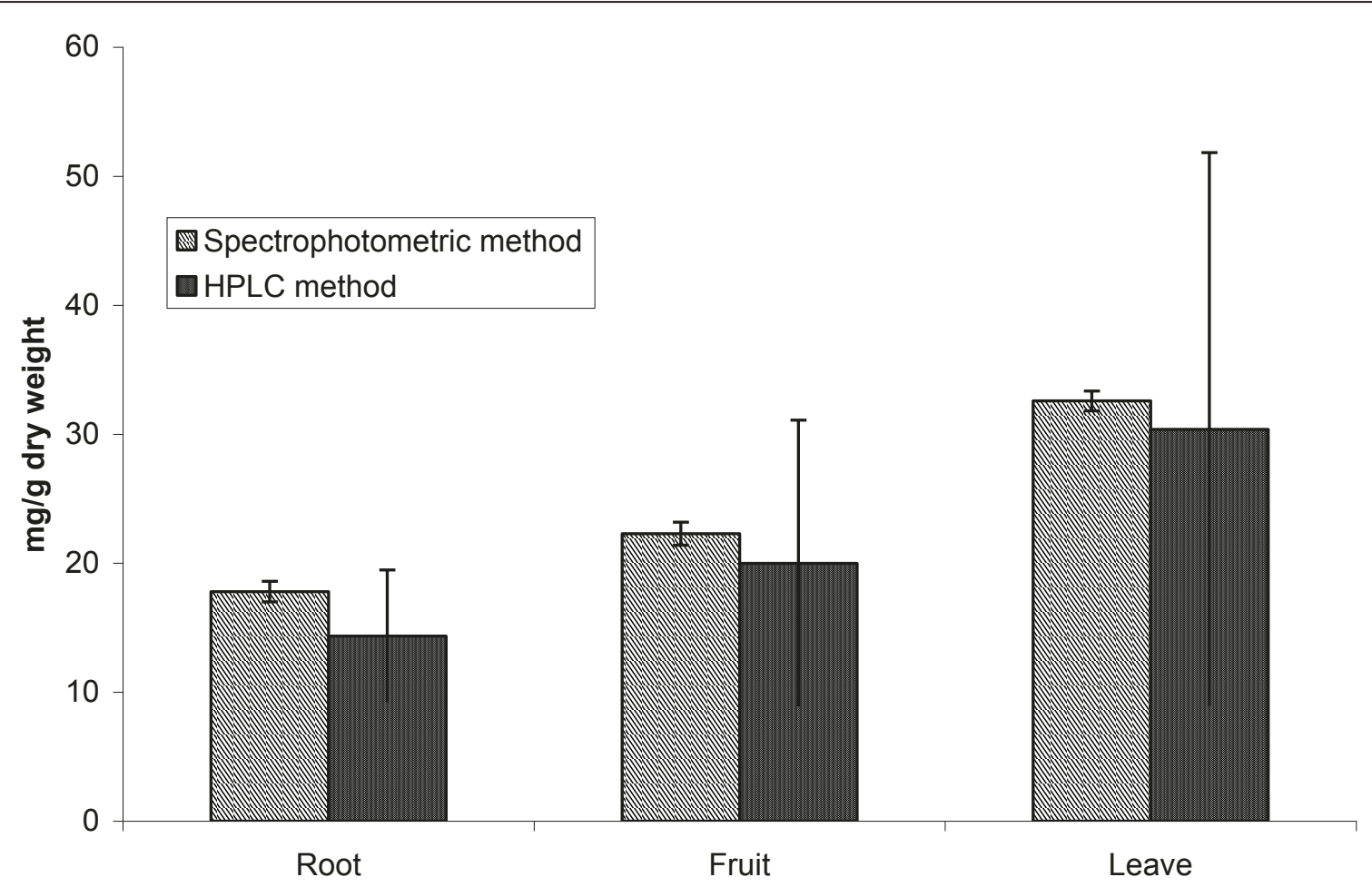

Figure 4 Comparison of total values of phenolic and flavonoid compounds ( $\mathrm{mg} / \mathrm{g}$ ) of $W$. somnifera roots, fruits and leaves obtained by spectrophotometric and HPLC method $(p<0.05)$. 
Table 3 Phenolic acids and flavonoids compounds detected in Withania somnifera roots, fruits and leaves using high performance liquid chromatography analysis.

\begin{tabular}{|c|c|c|c|c|c|c|}
\hline \multirow[t]{2}{*}{ SL No } & \multirow[t]{2}{*}{ Standard compounds } & \multirow[t]{2}{*}{ Retention time } & \multirow[t]{2}{*}{$\lambda_{\max }(\mathrm{nm})$} & \multicolumn{3}{|c|}{ Quantity of the identified compounds (mg/g DW) } \\
\hline & & & & Roots & Fruits & Leaves \\
\hline 1 & Catechin & 3.36 & 278 & 12.82 & 19.48 & 28.38 \\
\hline 2 & Gallic acid & 4.12 & 269,216 & ND & ND & 0.18 \\
\hline 3 & Syringic acid & 8.10 & 268,216 & ND & ND & 0.30 \\
\hline 4 & Vanillic acid & 8.61 & $224,249,269$ & ND & ND & 0.15 \\
\hline 5 & p-coumaric acid & 10.97 & $264,286,310$ & ND & ND & 0.80 \\
\hline 6 & Benzoic acid & 15.33 & 272,241 & 0.19 & ND & 0.80 \\
\hline 7 & Naringenin & 20.18 & $277,292,308$ & ND & 0.50 & ND \\
\hline 8 & Kaempferol & 25.59 & 361 & ND & 0.06 & ND \\
\hline \multicolumn{4}{|c|}{ Total phenolic compounds } & $13.01 \pm 8.93$ & $20.04 \pm 11.09$ & $30.61 \pm 11.41$ \\
\hline
\end{tabular}

$(\mathrm{ND}=$ Not detected), DW = dry weight

antioxidant activity, anti-ageing properties and cardiac health maintenance [35]. Catechins' beneficial effects are attributed to its ability to reduce oxidative stress, lipid peroxidation, free radical generation and unhealthy low density lipoprotein (LDL) cholesterol-oxidation [36]. There is also an evidence that suggests that catechins have a role in the protection against degenerative disorders [37]. Throughout the experiments, some catechins have also been shown to inhibit a key enzyme (squalene epoxidase) in the pathway of cholesterol biosynthesis [38]. The potent antioxidant properties of catechin reduce free radical damage to cells and prevent the oxidation of LDL cholesterol [39]. Besides catechin, other phenolic compounds found in the WSREt, WSFEt and WSLEt may also contribute to its medicinal and antioxidant properties [40]. Further studies to isolate individual active principles and antioxidant activity of individual extracts of roots, fruits as well as leaves through radical scavenging assay and their pharmacological validation in terms of modern medicine will be of great pharmacological importance in future which is under our consideration.

\section{Conclusion}

Five phenolics (gallic, syringic, benzoic, p-coumaric and vanillic acids) and three flavonoids (catechin, kaempferol, and naringenin) have been identified in WSREt, WSFEt and WSLEt and catechin was found in high concentrations especially in the leaves part, confirming the antioxidant potential and health benefits of $W$. somnifera.

\section{Acknowledgements}

This study was financially supported by Universiti Sains Malaysia RU grant (grant no. 1001/PPSP/8120201 and 1001/PPSP/815058). The authors would like to acknowledge the Department of Botany, Rajshahi University, Bangladesh and the Department of Pharmacology, School of Medical Sciences, Universiti Sains Malaysia for providing laboratory support and other facilities for this study.

\section{Author details}

${ }^{1}$ Department of Botany, Rajshahi University, Bangladesh. ${ }^{2}$ Department of Pharmacology, School of Medical Sciences, Universiti Sains Malaysia, 16150 Kubang Kerian, Kelantan, Malaysia. ${ }^{3}$ Human Genome Centre, School of Medical Sciences, Universiti Sains Malaysia, 16150 Kubang Kerian, Kelantan, Malaysia.

\section{Authors' contributions}

NA, MIK and MM have carried out the experimental parts of this investigation. MH, SAS and SHG supervised the work, evaluated the results and corrected the manuscript for publication. All authors read and approved the final manuscript.

\section{Competing interests}

The authors declare that they have no competing interests.

Received: 22 May 2011 Accepted: 19 August 2011

Published: 19 August 2011

\section{References}

1. Patwardhan B, Panse GT, Kulkarni PH: Ashwagandha a review. Journal of the National Integrated Medicine Association 1998, 30:7-11.

2. Dhuley JN: Adaptogenic and cardioprotective action of ashwagandha in rats and frogs. J Ethnopharmacol 2000, 70(1):57-63.

3. Agarwal R, Diwanay S, Patki P, Patwardhan B: Studies on immunomodulatory activity of Withania somnifera (ashwagandha) extracts in experimental immune inflammation. J Ethnopharmacol 1999, 67(1):27-35.

4. Prakash J, Gupta SK, Kochupillai V, Gupta YK, Joshi S: Chemopreventive activity of Withania somnifera in experimentally induced fibrosarcoma tumors in Swiss albino mice. Phytother Res 2001, 15(3):240-244.

5. Budhiraja RD, Sudhir S: Review of biological activity of withanolides. J Sci Ind Res 1987, 46:488-491.

6. Udayakumar R, Kasthurirengan S, Mariashibu TS, Rajesh M, Ramesh Anbazhagan V, Kim SC, Ganapathi A, Choi CW: Hypoglycaemic and hypolipidaemic effects of Withania somnifera root and leaf extracts on alloxan-induced diabetic rats. Int J Mol Sci 2009, 10:2367-2382.

7. Parthasarathy S, Santanam N, Ange N: Oxidised low-density lipoprotein, a two-faced Janus in coronary artery disease. Biochem Pharmacol 1998, 56:279-284.

8. Keller JN, Kindly MS, Holtberg FW, St Clair DK, Yen HC, Germeyer A, Steiner SM, Bruce-Keller AJ, Hutchins JB, Mattson MP: Mitochondrial manganese superoxide dismutase prevents neural apoptosis and reduces ischemic brain injury: suppression of peroxynitrite products, lipid peroxidation, and mitochondrial dysfunction. J Neurosci 1998, 18:687-697.

9. Perry G, Raine KA, Nunomura A, Watayc T, Sayre LM, Smith MA: How important is oxidative damage? Lessons from Alzheimer's disease. Free Radic Biol Med 2000, 28:831-834.

10. Kamat JP, Devasagayam TPA: Oxidative damage to mitochondria in normal and cancer tissues, and its modulation. Toxicology 2000, 155:73-82. 
11. Gorogawa SI, Kajimoto Y, Umayahara Y, Kaneto H, Watada H, Kuroda A, Kawamori D, Yasuda T, Matsuhisa M, Yamasaki Y, et al: Probucol preserves pancreatic-cell function through reduction of oxidative stress in type 2 diabetes. Diabetes Res Clin Pract 2002, 57:1-10

12. Hänninen $\mathrm{O}$, Kaartinen $\mathrm{K}$, Rauma AL, Nenenen M, Törrönen $\mathrm{R}$, Häkkinen $\mathrm{S}$, Adlercreutz $\mathrm{H}$, Laakso J: Antioxidants in vegan diet and rheumatic disorders. Journal of Toxicology 2000, 155:45-53.

13. Khodr B, Khalil Z: Modulation of inflammation by reactive oxygen species: implications for aging and tissue repair. Free Radic Biol Med 2001, 30:1-8.

14. Ferguson LR: Role of plant polyphenols in genomic stability. Mutat Res 2001, 475:89-111.

15. Netzel M, Strass G, Kaul C, Bitsch I, Dietrich H, Bitsch R: In vivo antioxidative capacity of composite berry juice. Food Res Int 2002, 35:213-216.

16. Zdunczyk Z, Frejnajel S, Wróblewska M, Juskiewicz J, Oszmianski J, Estrella I: Biological activity of polyphenols extracts from different plant sources. Food Res Int 2002, 35:183-186.

17. Tang S, Kerry JP, Sheehan D, Buckley DJ, Morrissey PA: Antioxidative effect of added tea catechins on susceptibility of cooked red meat, poultry and fish patties to lipid oxidation. Food Res Int 2002, 35:651-657.

18. Nuutila AM, Kammiovirta K, Caldentey KMO: Comparison of methods for the hydrolysis of flavonoids and phenolic acids from onion and spinach for HPLC analysis. Food Chem 2002, 76:519-525.

19. Shahrzad S, Bitsch I: Determination of some pharmacologically active phenolic acids in juices by high-performance liquid chromatography. J Chromatogr A 1996, 741:223-231.

20. Friend J: Phenolic compounds are believed to be an important part of the general defence mechanism of many plants. Phytochemisty soc 1985, 25:367-372.

21. Keefer JF, Schuster SM: Separation of citric acid cycle intermediates by high-performance liquid chromatography with ion pairing. J Chromatogr A 1986, 383(2):297-305

22. Scorska M, Matawska I, Gowniak K, Zgorka G: Qualitative and quantitative analysis of phenolic acids in Asclepias syriaca L. Acta Pol Pharm 2000, 57(1):69-72.

23. Kahkonen MP, Hopia Al, Vuorela HJPRJ, Pihlaja K, Kujala TS, Heinonen M: Antioxidant Activity of Plant Extracts Containing Phenolic Compounds. J Agric Food Chem 1999, 47:3954-3962.

24. Singleton VL, Orthofer R, Lamuela-Raventos RM: Analysis of total phenols and other oxidation substrates and antioxidant by means of FolinCiocalteu reagent. Meth Enzymol 1999, 299:152-178.

25. Zhishen J, Mengcheng T, Jianming W: The determination of flavonoid contents in mulberry and their scavenging effects on superoxide radicals. Food Chem 1999, 64:555-559.

26. Ferreira ICFR, Aires E, Barreira JCM, Estevinho LM: Antioxidant activity of Portuguese honey samples: Different contributions of the entire honey and phenolic extract. Food Chem 2009, 114:1438-1443.

27. Hatano T, Kagawa H, Yashura T, Okuda T: Two new flavonoids and other constituents in licorice root: Their relative astringency and radical scavenging effects. Chemical \& Pharmaceutical Bulletin 1988, 36:2090-2097.

28. Kaškonienè $\mathrm{V}$, Maruška A, Kornyšova O: Quantitative and qualitative determination of phenolic compounds in honey. Chemine Technologija 2009, 52(3):74-80.

29. Piljac-Žegarac J, Stipčević T, Belščak A: Antioxidant properties and phenolic content of different floral origin honeys. J ApiProd ApiMed Sci 2009, 1(2):43-50.

30. Udayakumar R, Kasthurirengan S, Vasudevan A, Mariashibu TS, Rayan JJS, Choi CW, Ganapathi A, Kim SC: Antioxidant Effect of Dietary Supplement Withania somnifera L. Reduce Blood Glucose Levels in Alloxan-Induced Diabetic Rats. Plant Foods Hum Nutr 2010, 65:91-98.

31. Escarpa A, González MC: Approach to the content of total extractable phenolic compounds from different food samples by comparison of chromatographic and spectrophotometric methods. Anal Chim Acta 2001, 427(1):119-127.

32. Azlim Almey AA, Ahmed Jalal Khan C, Syed Zahir I, Mustapha Suleiman K Aisyah MR, Kamarul Rahim K: Total phenolic content and primary antioxidant activity of methanolic and ethanolic extracts of aromatic plants' leafs. International Food Research Journal 2010, 17:1077-1084.

33. Liu SC, Lin JT, Wang CK, Chen HY, Yang DJ: Antioxidant properties of various solvent extracts from lychee (Litchi chinenesis Sonn.) flowers. Food Chem 2009, 114:577-581.
34. Shukla S, Mehtaa A, Johna J, Singha S, Mehtaa P, Vyasb SP: Antioxidant activity and total phenolic content of ethanolic extract of Caesalpinia bonducella seeds. Food Chem Toxicol 2009, 47(8):1848-1851.

35. Lambert JD, Yang CS: Cancer chemopreventive activity and bioavailability of tea and tea polyphenols. Mutat Res 2003, 523-524:201-208.

36. Coimbra S, Castro E, Rocha-Pereira P, Rebelo I, Rocha S, Santos-Silva A: The effect of green tea in oxidative stress. Clin Nutr 2006, 25:790-796.

37. Cooper R, Morré DJ, Morré DM: Medicinal benefits of green tea: part I. Review of noncancer health benefits. J Alternative Compl Med 2005, 11:521-528.

38. Abe I, Kashiwagi $Y$, Noguchi $H$, Tanaka T, Ikeshiro $Y$, Kashiwada $Y$ : Ellagitannins and hexahydroxydiphenoyl esters as inhibitor of vertebrate squalene epoxidase. J Nat Prod 2001, 64:1010-1014.

39. Cooper R, Morré DJ, Morré DM: Medicinal benefits of green tea: part II. Review of anticancer properties. J Alternative Compl Med 2005, 11:639-652.

40. Khalil MI, Sulaiman SA, Boukraa L: Antioxidant Properties of Honey and Its Role in Preventing Health Disorder. The Open Nutraceuticals Journal 2010, 3:6-16.

\section{Pre-publication history}

The pre-publication history for this paper can be accessed here: http://www.biomedcentral.com/1472-6882/11/65/prepub

doi:10.1186/1472-6882-11-65

Cite this article as: Alam et al:: High catechin concentrations detected in Withania somnifera (ashwagandha) by high performance liquid chromatography analysis. BMC Complementary and Alternative Medicine $201111: 65$

\section{Submit your next manuscript to BioMed Central and take full advantage of:}

- Convenient online submission

- Thorough peer review

- No space constraints or color figure charges

- Immediate publication on acceptance

- Inclusion in PubMed, CAS, Scopus and Google Scholar

- Research which is freely available for redistribution 